\title{
Comparison of Second-Generation Basal Insulin Analogs: A Review of the Evidence from Continuous Glucose Monitoring
}

\author{
Tadej Battelino, MD, Steven V. Edelman, $\mathrm{MD}^{2}{ }^{2}$ \\ Rimei Nishimura, $M D^{3}$ and Richard M. Bergenstal, MD ${ }^{4}$
}

\begin{abstract}
Many people with insulin-treated diabetes continue to experience inadequate glycemic control and a high incidence of hypoglycemic events, despite improvements in therapeutic strategies. While glycated hemoglobin $\left(\mathrm{HbA}_{1 c}\right)$ is currently recognized as the gold-standard for assessing glycemic control, the measure reflects mean blood glucose levels over a period of time, does not inform on acute glycemic deviations, and can be unreliable in certain populations. Continuous glucose monitoring (CGM) facilitates the acquisition of blood glucose data around the clock and, importantly, predicts and/or captures acute hyper- and hypoglycemic episodes. In light of the recent publication of the Time in Range (TIR) International Consensus Group report on key CGM metrics, we performed a review of current CGM evidence for second-generation basal insulins in both people with type 1 and type 2 diabetes. The identified studies highlight the varied CGM-related metrics used to assess basal insulins, which complicate comparisons. Furthermore, all studies had small sample sizes and typically were of short duration, which may account for the lack of statistically significant between-treatment differences observed. Differences were seen in the titration approaches used and the settings in which participants were observed. These results highlight the need for further studies of secondgeneration basal insulin analogs that are designed to capture the standard metrics proposed by the TIR consensus group, with additional consideration given to sample size and study duration.
\end{abstract}

Keywords: Continuous glucose monitoring, Basal insulins, Insulin therapy, Time in Range.

\section{Introduction}

D ESPITE IMPROVEMENTS IN THERAPY, many people with insulin-treated diabetes continue to experience inadequate glycemic control and a high incidence of hypoglycemic events. ${ }^{1-4}$ In both people with type 1 diabetes (T1D) and type 2 diabetes (T2D), suboptimal management of blood glucose is associated with a variety of micro- and macrovascular complications as well as hyperglycemia, diabetic ketoacidosis, and hypoglycemia. ${ }^{5-1}$

Glycated hemoglobin $\left(\mathrm{HbA}_{1 \mathrm{c}}\right)$ is recognized as the goldstandard for assessing glycemic control. ${ }^{8,9}$ However, while
$\mathrm{HbA}_{1 \mathrm{c}}$ provides a surrogate marker for average blood glucose, it may not provide a true representation of glycemic variability, including acute hyper- and hypoglycemic episodes. ${ }^{8}$ The accuracy of $\mathrm{HbA}_{1 \mathrm{c}}$ measurement can also be compromised by conditions and drugs that affect red blood cell turnover, such as untreated iron deficiency, hemolysis and splenomegaly, and erythropoietin, iron, and dapsone treatment, respectively. ${ }^{9}$ Continuous glucose monitoring (CGM), which includes both real-time or intermittently scanned CGM, provides comprehensive data on 24-h blood glucose levels and can capture acute glycemic excursions. ${ }^{8}$ However, its use in clinical practice and randomized

${ }^{1}$ UMC-University Children's Hospital and Faculty of Medicine, University of Ljubljana, Ljubljana, Slovenia.

${ }^{2}$ University of California, San Diego, California, USA.

${ }^{3}$ The Jikei University School of Medicine, Tokyo, Japan.

${ }^{4}$ International Diabetes Center Park Nicollet, Minneapolis, Minnesota, USA.

(C) Tadej Battelino, et al., 2020; Published by Mary Ann Liebert, Inc. This Open Access article is distributed under the terms of the Creative Commons License (http://creativecommons.org/licenses/by/4.0), which permits unrestricted use, distribution, and reproduction in any medium, provided the original work is properly credited. 
controlled trials (RCTs) has so far been limited by the lack of consensus on appropriate glycemic targets for individuals with diabetes and how data from CGM should be assessed. ${ }^{8}$ However, it should be noted that, while $\mathrm{HbA}_{1 \mathrm{c}}$ levels have been shown to be associated with microvascular complications, ${ }^{10}$ no similar prospective data are yet available for CGM-based metrics.

In 2019, the Advanced Technologies and Treatments for Diabetes (ATTD) Congress convened an international panel of people with diabetes, clinicians, and researchers to develop practical, evidence-based CGM metrics and targets that can be applied in both trials and routine care settings; the Time in Range [TIR] International Consensus report. ${ }^{8}$ The group recommended key CGM metrics that provide information and clinical targets for diabetes treatment: number of days CGM worn (14 days recommended), percentage of time CGM is active ( $70 \%$ over 14 days or 10 full days recommended), mean glucose, glucose management indicator (replaces previous estimated $\mathrm{HbA}_{1 \mathrm{c}}{ }^{11}$ ), glycemic variability (coefficient of variation $[\mathrm{CV}]$, recommended target $\leq 36 \%$ ), and time in glucose ranges (TIR, time below range [TBR; hypoglycemia], and time above range [TAR; hyperglycemia]). Recommended targets for assessment of glycemic control are shown in Figure $1 .{ }^{8}$ Utilizing these targets and metrics in clinical studies and routine practice can complement traditional $\mathrm{HbA}_{1 \mathrm{c}}$ values in data analysis and facilitate informed decision-making. To further assist with clinical implementation, the consensus group also recommended a standardized CGM report, for example, the Ambulatory Glucose Profile, that incorporates the aforementioned CGM metrics and targets into a succinct single-page summary. ${ }^{8}$

The second-generation basal insulins, insulin glargine $300 \mathrm{U} / \mathrm{mL}$ (Gla-300), and insulin degludec (IDeg) have longer durations of action and smoother pharmacokinetic/pharmacodynamic (PK/PD) profiles compared with first-generation insulins, such as insulin glargine $100 \mathrm{U} / \mathrm{mL}$ (Gla-100) or insulin detemir (IDet), with low within-day fluctuation and betweenday variability. ${ }^{12-15}$ Head-to-head comparisons between Gla-300 and IDeg in people with T1D are limited, with two PK/PD glycemic clamp studies directly comparing Gla-300 and IDeg at steady-state in people with T1D reporting conflicting results. ${ }^{16,17}$

Furthermore, two head-to-head RCTs have compared the efficacy and safety of Gla-300 and IDeg in individuals with T2D and have also showed conflicting results: BRIGHT (insulin-naive) and CONCLUDE (insulin pretreated). BRIGHT demonstrated noninferiority of Gla-300 versus IDeg $(100 \mathrm{U} / \mathrm{mL})$ in $\mathrm{HbA}_{1 \mathrm{c}}$ reductions over the full 24 weeks (primary endpoint). In addition, with the exception of a lower anytime confirmed hypoglycemia event rate with Gla-300 compared with IDeg in the titration period, clinical outcomes data showed more similarities than differences between the two analogs. ${ }^{18}$ CONCLUDE did not meet its primary endpoint (superiority of IDeg [200 U/mL] vs. Gla-300 for events of confirmed [ $<56 \mathrm{mg} / \mathrm{dL}[<3.1 \mathrm{mmol} / \mathrm{L}]]$ or severe hypoglycemia during a 36-week maintenance phase 2 period). ${ }^{19,20}$ Exploratory secondary analyses showed lower rates of nocturnal symptomatic hypoglycemia and severe hypoglycemia with IDeg versus Gla-300, ${ }^{20}$ but these analyses cannot be considered to be conclusive. ${ }^{21}$ However, both BRIGHT and CONCLUDE utilized traditional $\mathrm{HbA}_{1 \mathrm{c}}$ measurements, alongside self-monitored blood glucose (SMBG) measurements and laboratory-measured FPG to compare glycemic outcomes. ${ }^{18,22}$ To date, no head-to-head trials comparing the efficacy and safety of these two second-generation basal insulins utilizing CGM have been conducted in people with T1D.

\section{Blood glucose threshold Recommended time in ranges*}

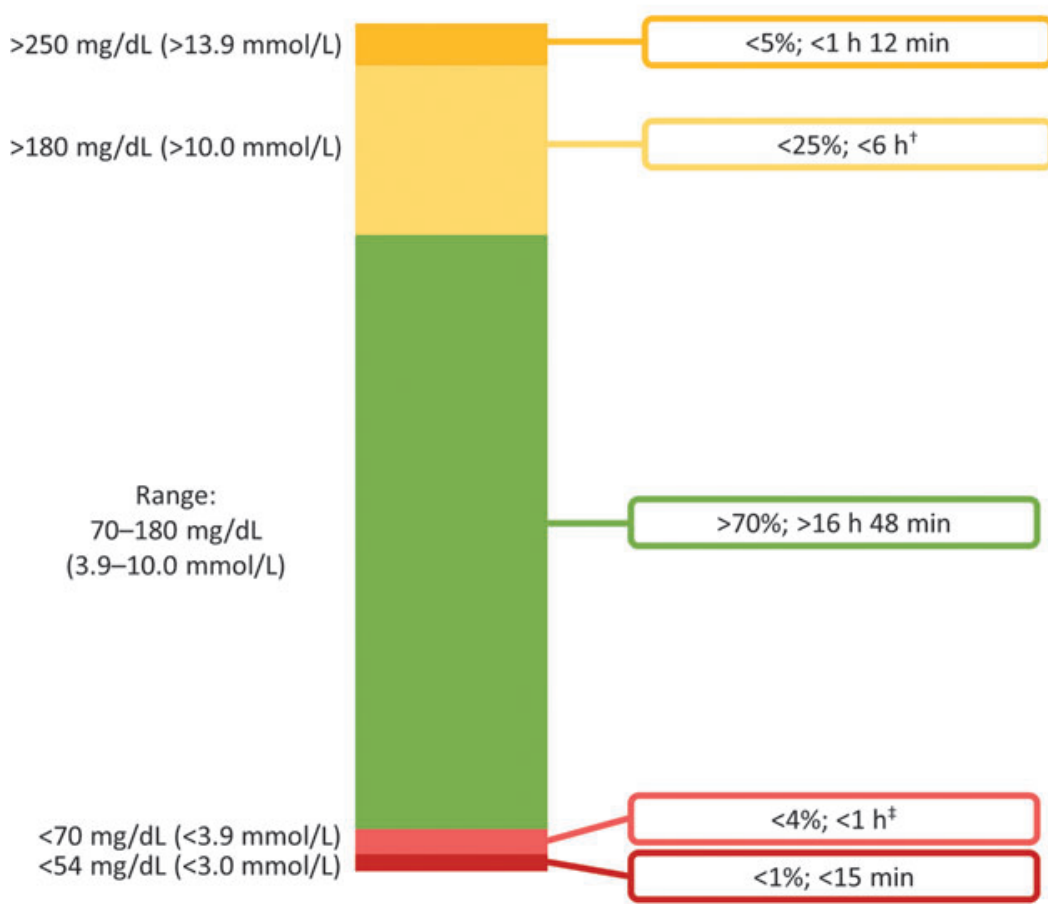

FIG. 1. CGM-based targets for most individuals with T1D or T2D (adapted from Battelino et al. $)^{8}$ The diagram shows recommended TIR, TAR, and TBR targets for most individuals with T1D or T2D; however, targets for older individuals, individuals with T1D <25 years; individuals with T1D who are pregnant or individuals with gestational diabetes vary slightly from those shown. *Percentage values demonstrate the recommended percentage of a $24 \mathrm{~h}$ period within each range; ${ }^{\dagger}$ Includes the percentage of values $>250 \mathrm{mg} / \mathrm{dL}(>13.9 \mathrm{mmol} / \mathrm{L})$; ${ }^{*} \mathrm{In}-$ cludes the percentage of values $<54 \mathrm{mg} / \mathrm{dL}$ ( $<3.0 \mathrm{mmol} / \mathrm{L})$. CGM, continuous glucose monitoring; T1D, type 1 diabetes; T2D, type 2 diabetes; TAR, time above range; TBR, time below range; TIR, time in range. American Diabetes Association. Clinical targets for continuous glucose monitoring data interpretation: recommendations from the international consensus on time in range, American Diabetes Association, 2019. ${ }^{8}$ Copyright and all rights reserved. Material from this publication has been used with the permission of American Diabetes Association. 
Even with second-generation basal insulin analogs, clinically relevant hypoglycemia still occurs ${ }^{3}$; it is therefore imperative that glycemic control is assessed in detail. How glucose data are used to compare basal insulin therapies is currently under scrutiny, ${ }^{23}$ and CGM data will contribute further to such discussion. ${ }^{8,24}$ CGM data offer the prospect of exploring the glycemic profile of basal insulin in more depth than $\mathrm{HbA}_{1 \mathrm{c}}$ data, including measures of glucose variability, glucose TIR, TBR, and TAR. Therefore, the aim of this review is to examine the quality of current CGM evidence for the second-generation basal insulins Gla-300 and IDeg in people with T1D and T2D. Where possible, metrics from the ATTD 2019 consensus guidelines have been used to facilitate comparisons.

\section{Search Strategy and Selection Criteria}

References for this review were identified through searches of PubMed for articles published from January 1, 2000, to May 7, 2019, using the terms "CGM[Title/abstract] OR (continuous[Title/abstract] AND glucose[Title/abstract] AND monitor*[Title/abstract]) AND (degludec[Title/abstract])", and "CGM[Title/abstract] OR (continuous[Title/abstract] AND glucose[Title/abstract] AND monitor*[Title/abstract]) AND (Gla-300[Title/abstract] OR (glargine[Title/abstract] AND 300[Title/abstract]))." Searches identified 42 articles which were subsequently screened for relevance and to remove duplicates. Articles not published in English, not providing CGM-metrics, or comparing fixed-ratio combinations of basal insulins with a glucose-like 1 receptor agonist were excluded resulting in 17 relevant articles.

\section{CGM Findings for Second-Generation Basal Insulin Analogs}

The results of studies utilizing CGM to compare clinical outcomes with first- and second-generation basal insulin analogs are reported below. It is important to note, however, that these studies were conducted before the recent development of the TIR International Consensus Report; as such, there is considerable diversity in the reporting of key CGM metrics.

\section{CGM Data Comparing First- and Second-Generation Basal Insulin Analogs}

\section{Gla-300 versus Gla-100}

Three trials were identified that compared Gla-300 and Gla-100 using CGM. ${ }^{25-27}$ The U.S. CGM study was the first large trial in people with T1D with CGM endpoints directly comparing first- and second-generation basal insulin analogs, and showed that TIR and variability results for Gla-300 and Gla-100 were similar (Table 1). ${ }^{25}$ In the study by Okajima et al., nocturnal hypoglycemia was significantly lower for Gla-300 versus Gla-100, ${ }^{27}$ and there were numerical differences in favor of Gla-300 in the other two studies (Table 1). ${ }^{25,26}$ However, because different metrics were used in the studies that reported this endpoint, any comparisons of variability outcomes across trials should be treated with caution. Bergenstal et al. used SMBG and CGM to assess hypoglycemia, with betweentreatment comparisons only provided for SMBG results, while Okajima et al. used a CGM-measured blood glucose of $<70 \mathrm{mg} / \mathrm{dL}$, although hypoglycemia was reported as "frequency" of events. ${ }^{25,27}$ Jinnouchi et al. did not report the method used to analyze hypoglycemic events. ${ }^{26}$

\section{IDeg versus Gla-100 or IDet}

TIR outcomes were provided in two trials comparing the second-generation basal insulin analog IDeg with firstgeneration basal insulin analogs Gla-100 or IDet; however, neither trial reported TIR differences between IDeg and Gla$100 .^{28,29}$ Based on the percentage $\mathrm{CV}$ data, which is the variability outcome recommended by the ATTD consensus panel, ${ }^{8}$ there did not appear to be any differences in glucose variability between IDeg and Gla-100 or IDet in the three trials that reported this outcome overall (Table 1). ${ }^{28,30,45} \mathrm{~A}$ fourth trial split participants into those with high glycemic variability (HGV) and low glycemic variability (LGV), based on a CV threshold of $34 \%$ (similar to the threshold of $\leq 36 \%$ recommended by the ATTD 2019 consensus group $\left.{ }^{8}\right) .{ }^{31}$ In the HGV group, switching from either Gla-100 or IDet to IDeg was associated with a significant reduction in glucose variability at 12 weeks (mean [SD]: baseline, $44.7 \%$ [7.3] vs. week 12, 33.6\% [10.1]; $P<0.001)$. This significant reduction was not observed in the LGV group. ${ }^{31}$ It should be noted that people with T1D were a minority in this study $(27.6 \%)$. Nevertheless, these data suggest that, compared with first-generation basal insulins, IDeg may be of benefit for individuals with high glycemic fluctuations; this may be of clinical importance for individuals with T1D.

One of the six trials that reported glucose variability as SD reported a significant difference between Gla-100 and IDeg (mean [SD]: $51.6 \mathrm{mg} / \mathrm{dL}$ [18.1] vs. $43.7 \mathrm{mg} / \mathrm{dL}$ [13.7], respectively; $P=0.031){ }^{32}$ The other five trials that reported SD glucose variability identified no significant differences between IDeg and Gla-100 or IDet.

TBR for the blood glucose thresholds of both $<55$ and $<70 \mathrm{mg} / \mathrm{dL}$ were reported in one study, and $<70 \mathrm{mg} / \mathrm{dL}$ alone in six studies (Table 1). Galasso et al. reported a significantly reduced TBR at week 12 following a switch from twice-daily Gla-100 or IDet to IDeg $(<55 \mathrm{mg} / \mathrm{dL}, P=0.02$ and $<70 \mathrm{mg} / \mathrm{dL}, P=0.05) .{ }^{28} \mathrm{TBR}<70 \mathrm{mg} / \mathrm{dL}$ was generally not significantly different between Gla-100/IDet and IDeg in the other trials; only one of the six trials found a significant reduction in mean anytime TBR between treatment with Gla100 or IDet at baseline and after 12 weeks of treatment with IDeg (mean [SD]: $14.7 \%$ [14.9] vs. 9.6\% [11.5]; $P=0.045){ }^{30}$ The remaining trials did not report TBR data.

Although not identified with the search criteria, as CGM was not mentioned in the title or abstract, the BEGIN Young trial comparing IDeg and IDet in children (1-17 years of age) with T1D published limited CGM data from a small subset of participants. These data showed no significant differences in the number or duration of hypoglycemic or hyperglycemic events both at any time of day or during the night. ${ }^{33}$ Further unpublished CGM data are also available from the clinical study reports of the BEGIN T1D trial and the BEGIN Once Long, and BEGIN FLEX trials in people with T2D. ${ }^{34-36}$ No statistical differences were observed between Gla-100 and IDeg in variability $(\mathrm{CV}, \%)$ of anytime or nocturnal glucose profiles or time in hypoglycemia (several blood glucose thresholds were used, although reported data were limited). ${ }^{34-36}$ It is interesting to note that while PK/PD studies show substantially lower PD variability with IDeg than Gla-100 in T1D, ${ }^{14}$ this is generally 


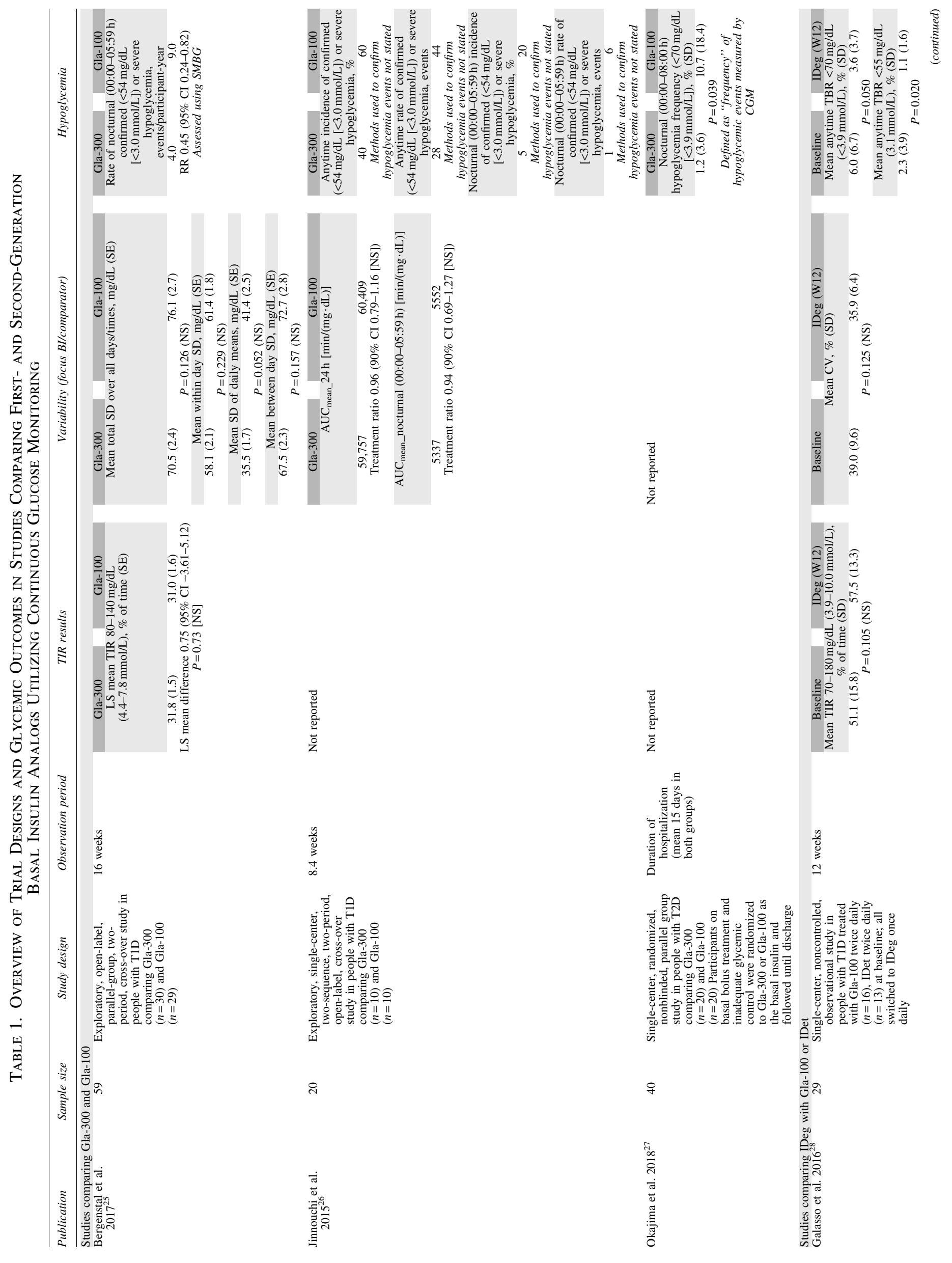




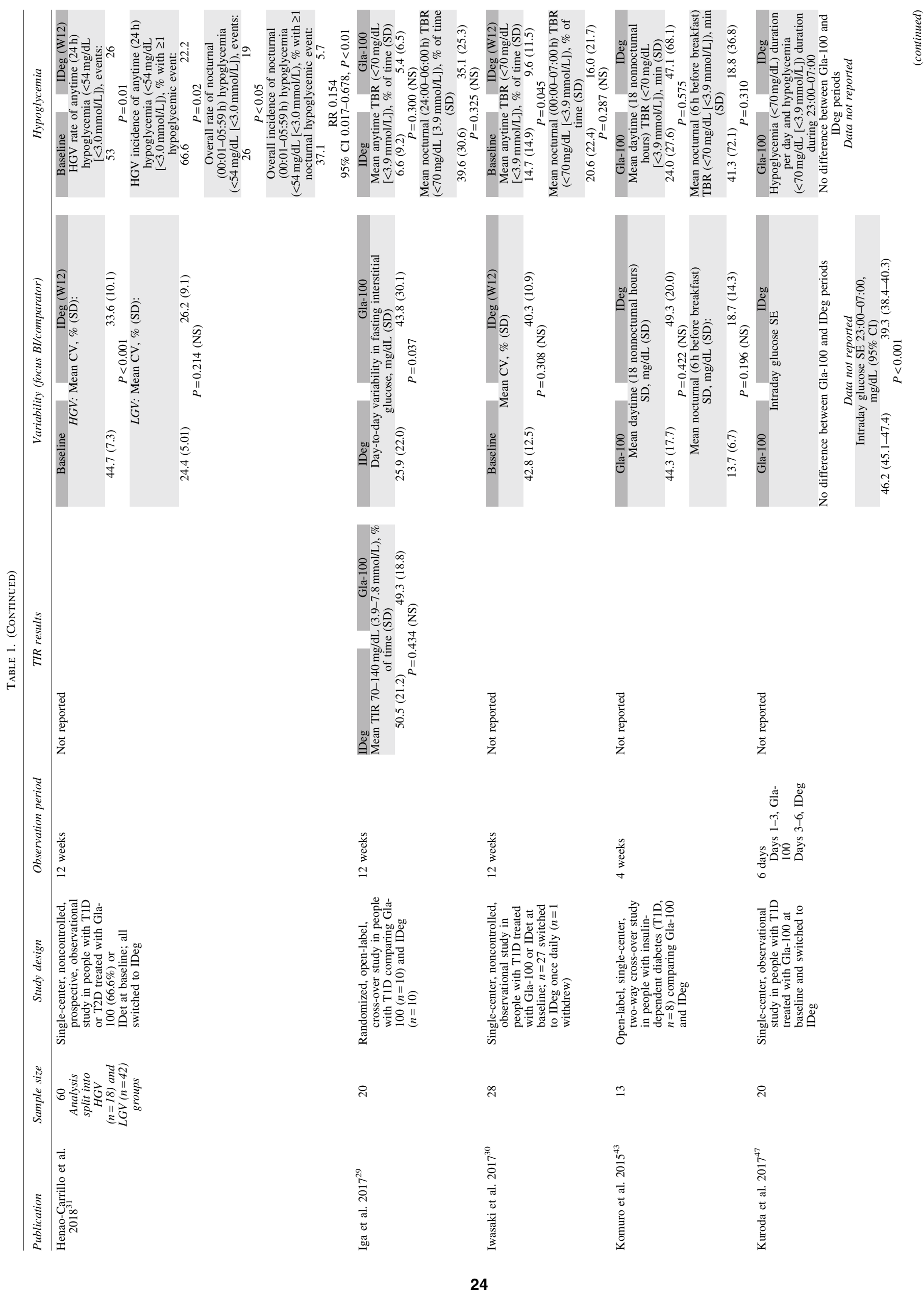




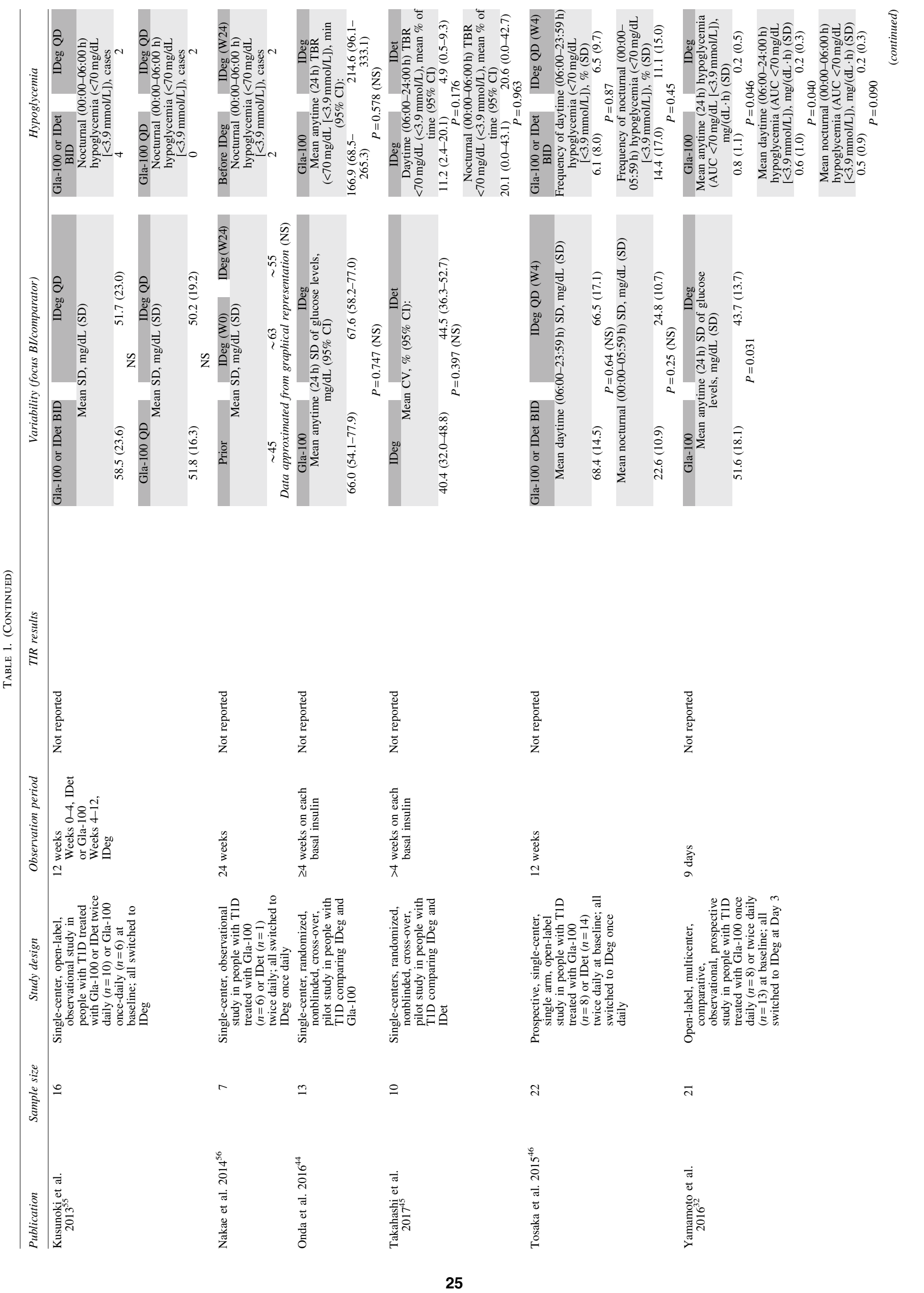




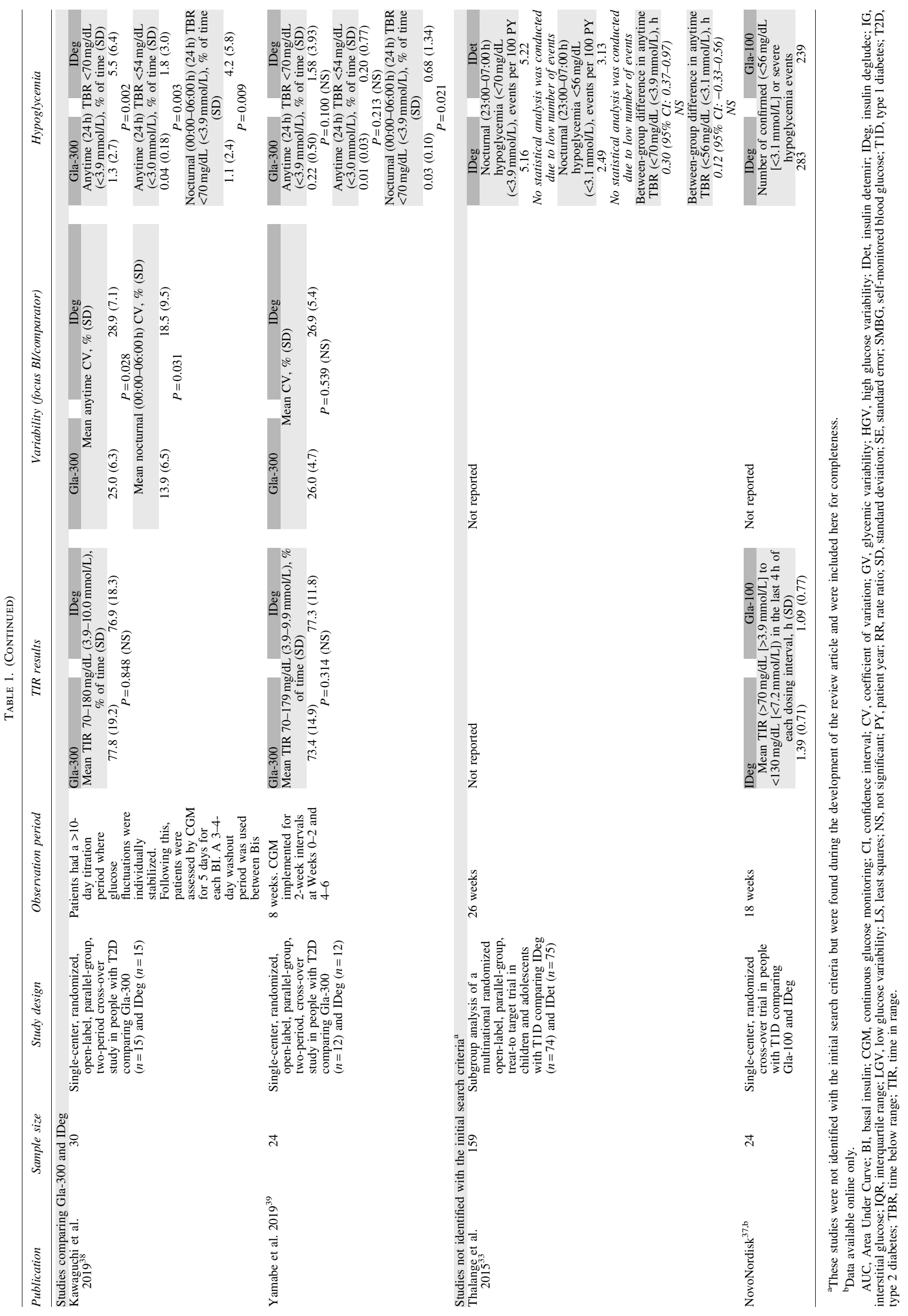


not confirmed by these limited CGM data. A further trial (NCT01569841) that was not identified in the original search involved 24 individuals with T1D treated with either Gla-100 or IDeg in an 18-week randomized cross-over trial. This study comprised two 6-week treatment periods, in which patients were either treated with IDeg or Gla-100, with their basal insulins switched between treatment periods; CGM measurements were taken in the last 2 weeks of each treatment period. ${ }^{37}$ Although no statistical comparisons were made between treatment groups, TIR was shorter, but fewer confirmed ( $<56 \mathrm{mg} / \mathrm{dL}[<3.1 \mathrm{mmol} / \mathrm{L}])$ hypoglycemia events were seen with Gla-100 versus IDeg. ${ }^{37}$

\section{CGM Data Comparing the Second-Generation Basal Insulin Analogs Gla-300 and IDeg}

Two trials comparing Gla-300 and IDeg were identified. Both were recently published single-center, open-label, parallel-group, cross-over studies in people with T2D, conducted in Japan. ${ }^{38,39}$ Glycemic control was similar between insulins in both studies (Table 1), with both insulins showing TIR values over the $70 \%$ recommended by the ATTD panel. ${ }^{8}$ Anytime variability $(\mathrm{CV}, \%)$ was similar or lower with Gla300 versus IDeg in both studies. Kawaguchi et al. indicated that overnight glucose variability favored Gla-300 over IDeg; $\mathrm{CV}$ between 00.00 and $06.00 \mathrm{~h}$ was lower for Gla-300 versus IDeg (mean [SD]: $13.9 \%$ [6.5] vs. $18.5 \%$ [9.5], respectively; $P=0.031){ }^{38}$ Although these results contrast with the lower within-day variability reported for IDeg versus Gla-300 in the previously mentioned Heise et al. PK/PD clamp study, ${ }^{16}$ the differences between the study populations of Heise et al. and the two Japanese studies make comparison very difficult. Measures of mean time in hypoglycemia also favored Gla-300 over IDeg, with significantly less nocturnal hypoglycemia (TBR $<70 \mathrm{mg} / \mathrm{dL}[<3.9 \mathrm{mmol} / \mathrm{L}]$ ) in both studies (Kawaguchi et al.: Gla-300, 1.1 [2.4]\% of time vs. IDeg, 4.2 [5.8]\% of time, $P=0.009$; Yamabe et al.: Gla-300, $0.03[0.10] \%$ of time vs. IDeg 0.68 [1.34]\% of time, $P=0.021$; Table 1). ${ }^{38,39}$

\section{Gla-300 versus IDeg in T1D: The InRange Study}

There is currently a paucity of large CGM studies comparing the two second-generation basal insulin analogs; however, the InRange study aims to address this. InRange (NCT04075513) is a 12-week, randomized, open-label, twoarm, parallel group, multicenter study that uses CGM to compare Gla-300 with IDeg in 340 adult participants with T1D. ${ }^{40}$ In line with the recent TIR International Consensus Group report, ${ }^{8}$ which highlights TIR as the most important CGM metric, the primary endpoint of InRange is percent time spent in the glucose range of $\geq 70$ to $\leq 180 \mathrm{mg} / \mathrm{dL}(\geq 3.9$ to $\leq 10 \mathrm{mmol} / \mathrm{L}$ ) at week 12 . ${ }^{40}$

\section{Conclusions}

CGM can provide a powerful tool in RCTs studying the efficacy and safety of second-generation basal insulin analogs as it can provide comprehensive data on 24-h blood glucose levels. ${ }^{8}$ In this regard, evidence on day-to-day and within-day glycemic variability and acute glycemic excursions may be better captured with CGM compared with $\mathrm{HbA}_{1 \mathrm{c}}$ and SMBG measurements. Furthermore, secondgeneration basal insulins have a lower hypoglycemia risk than first-generation basal insulins ${ }^{41,42}$ and comparisons of hypoglycemia risk between second-generation basal insulins may be limited by small numbers of events, particularly of serious hypoglycemia $(<54 \mathrm{mg} / \mathrm{dL}[<3.0 \mathrm{mmol} / \mathrm{L}]) ; \mathrm{CGM}$ offers the potential to facilitate such comparisons by ensuring that all glycemic excursions are captured.

However, CGM data on the efficacy and safety of secondgeneration basal insulins are currently limited; indeed, the present analysis only identified 19 such studies. Comparisons across studies utilizing CGM are further limited by a lack of consistency in the metrics used to assess outcomes in such trials, as highlighted by the substantial diversity observed across studies included in the present analysis. Other differences between studies included the titration methods used, including the FPG targets used and the titration approaches with some studies allowing titration throughout, ${ }^{26,39}$ while others did not perform any adjustments to basal insulin doses ${ }^{43}$ and some performed CGM once FPG targets had been reached following a titration period. ${ }^{32,38}$ In addition, the settings in which participants were monitored varied across studies, with some participants admitted in a hospital setting to control for the potential influence of diet and exercise, ${ }^{27,32}$ while others were conducted in an "outpatient" setting. ${ }^{39,44-46}$ The majority of studies identified were also of short duration, with some studies being only 6-15 days long ${ }^{27,32,47}$ and the majority being between 8 and 12 weeks long; this period of time will likely not enable sufficient data on hypoglycemia events to be captured to enable statistical comparisons and is not long enough to fully explore the efficacy and safety profile of basal insulins, particularly during the maintenance phase of treatment, where minimal dose adjustments are made. Further limitations of CGM studies identified in the present analysis included the small population sizes (with most direct comparison studies having fewer than 30 participants in each treatment arm), or with sample sizes being derived from subpopulations of larger RCTs, such as the BEGIN YOUNG study; thus these studies may have had insufficient statistical power to detect key outcomes. $^{33}$

It is therefore evident that there is a need for future RCTs utilizing CGM for head-to-head comparisons of secondgeneration basal insulins, or for comparisons with firstgeneration basal insulins. Such studies would utilize the TIR Consensus Group recommendations to ensure uniformity of CGM metrics data interpretation across studies. ${ }^{8}$ Additional considerations would be the use of evidence-based titration protocols and longer study durations to ensure that data are captured both during active titration of investigational basal insulins and during the "maintenance phase," where minimal dosage adjustments are performed. The InRange trial, with its relatively large population and CGM-derived metrics for the primary outcome, will help to provide further evidence for efficacy and safety of head-to-head comparisons of the two second-generation basal insulin analogs, Gla-300 and IDeg. ${ }^{40}$

In addition to the potential of CGM for assessment of basal insulins in a trial setting, CGM has also demonstrated significant benefits in the treatment of people with T1D, or with T2D requiring intensive insulin treatment, and is recommended by a number of medical societies. ${ }^{4-52}$ The customizable TIR metric obtained using CGM provides a valuable, streamlined measure of glycemic control that can be actively used for daily therapeutic decision-making in 
clinical practice and has the potential to allow the specific needs of each individual with diabetes to be addressed, including those with gestational diabetes or at high risk, and those for whom $\mathrm{HbA}_{1 \mathrm{c}}$ measurement may be unreliable. ${ }^{8}$ However, its use outside of the clinical trial setting has so far been limited, largely due to the previous lack of reimbursement for such devices in addition to a lack of consensus for targets, metrics, and standardized CGM data reports. However, a recent prospective observational study, which explored the effectiveness of CGM in 900 individuals following introduction of NHS-funding for CGM, demonstrated significant reductions in $\mathrm{HbA}_{1 \mathrm{c}}$ (median change from last value before CGM use and most recent value: $-0.4 \%$ [interquartile range: -0.9 to 0 ]; $P<0.001)$ in addition to significant reductions in hospital admissions for diabetic ketoacidosis $(P=0.043) .{ }^{53}$ In addition, the FUTURE study, a large real-world observational study in Belgium, showed that after CGM was approved for reimbursement, there were significant reductions in hospitalizations due to hypoglycemia or diabetic ketoacidosis and reduced absenteeism, compared with the previous year. ${ }^{54}$ Adoption of the recent ATTD consensus guidelines ${ }^{8}$ will hopefully facilitate the use of CGM, which, in turn, could improve outcomes for people with diabetes.

Furthermore, real-world evidence studies and registries that collect CGM metrics would be valuable sources of data on the effectiveness and safety of second-generation basal insulins in a real-life clinical practice setting. In particular, such studies could provide data from vulnerable populations who are often excluded from RCTs, such as children, pregnant women with T1D, T2D or gestational diabetes, and older individuals.

While the present analysis provides an overview of the current literature on CGM studies of second-generation basal insulins, the search methodology used may have not identified some relevant reports, as evidenced by the postsearch identification of two additional reports that have been included.

In summary, CGM has potential as a valuable tool for assessment of second-generation basal insulins in both randomized trials and real-world studies. However, current literature of CGM-related studies of second-generation basal insulins are limited by the varied metrics used, the small population sizes and short study durations. Future studies comparing second-generation basal insulins are needed that utilize the TIR Consensus Group recommended metrics ${ }^{8}$ to provide uniformity in reporting, are adequately powered to provide meaningful statistical comparisons and are of sufficient duration to provide evidence of safety and efficacy in the titration and maintenance phases of insulin use.

\section{Authors' Contributions}

T.B.: Contributed to the concept and design of the review, provided comments and input to all drafts of the article and interpretation of the results and had responsibility for approving the final version for submission. S.E.: Provided comments and input to all drafts of the article and interpretation of the results and had responsibility for approving the final version for submission. R.N.: Provided comments and input to all drafts of the article and interpretation of the results and had responsibility for approving the final version for submission. R.M.B.: Provided comments and input to all drafts of the article and interpretation of the results and had responsibility for approving the final version for submission.

\section{Author Disclosure Statement}

T.B. has received honoraria for participation on advisory boards for Novo Nordisk, Sanofi, Eli Lilly, Boehringer, Medtronic, and Bayer HealthCare and as a speaker for AstraZeneca, Eli Lilly, Bayer, Novo Nordisk, Medtronic, Sanofi, and Roche; owns stocks of DreaMed Diabetes; T.B.'s institution has received research grant support and travel expenses from Abbott Diabetes Care, Medtronic, Novo Nordisk, GluSense, Sanofi, Sandoz, and Diamyd. S.E.: Board member: Senseonics; Speakers bureau and advisory panel: AstraZeneca, Dexcom, Eli Lilly, MannKind, Merck, Novo Nordisk, Sanofi. R.N. has received research support from Japan Diabetes Foundation, Boehringer Ingelheim, Daiichi-Sankyo and Astellas; has participated in speakers bureau/advisory panels for Novo Nordisk, Eli Lilly, Sanofi, Kissei, Astellas, Boehringer Ingelheim, Daiichi-Sankyo, Tanabe-Mitsubishi, AstraZeneca, Kowa, Ono, Johnson \& Johnson, Medtronic and Takeda; and served as a consultant for Boehringer Ingelheim and Eli Lilly. R.B. has received research support from, consulted for, or has been on a scientific advisory board for Abbott Diabetes Care, Dexcom, Eli Lilly, Johnson \& Johnson, Medtronic, Novo Nordisk, Onduo, Roche, Sanofi, and United HealthCare. His research is partly funded by the National Institute of Diabetes and Digestive and Kidney Diseases (National Institutes of Health grant DK108611). R.M.B.'s employer, the nonprofit HealthPartners Institute, contracts for his services and no personal income goes to R.M.B.

\section{Funding Information}

Editorial assistance was provided by Hannah Brown, $\mathrm{PhD}$, of Fishawack Communications Ltd., whose service was funded by Sanofi. The DELIVER studies were funded by Sanofi. All authors take complete responsibility for the interpretation of the data in this review.

\section{References}

1. Emral R, Pathan F, Cortes CAY, et al. Self-reported hypoglycemia in insulin-treated patients with diabetes: results from an international survey on 7289 patients from nine countries. Diabetes Res Clin Pract 2017;134:17-28.

2. Khunti K, Alsifri S, Aronson R, et al. Rates and predictors of hypoglycaemia in 27585 people from 24 countries with insulin-treated type 1 and type 2 diabetes: the global HAT study. Diabetes Obes Metab 2016;18:907-915.

3. Foster NC, Beck RW, Miller KM, et al. State of type 1 diabetes management and outcomes from the T1D exchange in 2016-2018. Diabetes Technol Ther 2019;21: 66-72.

4. Pettus JH, Zhou FL, Shepherd L, et al. Incidences of severe hypoglycemia and diabetic ketoacidosis and prevalence of microvascular complications stratified by age and glycemic control in U.S. adult patients with type 1 diabetes: a realworld study. Diabetes Care 2019:dc190830.

5. Fowler MJ. Microvascular and macrovascular complications of diabetes. Clin Diabetes 2008;26:77-82.

6. Herman WH, Braffett $\mathrm{BH}$, Kuo S, et al. What are the clinical, quality-of-life, and cost consequences of 30 years of excellent vs. poor glycemic control in type 1 diabetes? J Diabetes Complications 2018;32:911-915.

7. International Diabetes Federation. IDF Diabetes Atlas, $8^{\text {th }}$ ed. 2017. Brussels, Belgium, 2017. Available at: https:// 
www.idf.org/e-library/epidemiology-research/diabetesatlas/134-idf-diabetes-atlas-8th-edition.html. (accessed 3 September 2020).

8. Battelino T, Danne T, Bergenstal RM, et al. Clinical targets for continuous glucose monitoring data interpretation: recommendations from the international consensus on time in range. Diabetes Care 2019:dci190028.

9. Wright LA, Hirsch IB. Metrics beyond hemoglobin A1C in diabetes management: time in range, hypoglycemia, and other parameters. Diabetes Technol Ther 2017;19(S2):S16S26.

10. Nathan DM, Genuth S, Lachin J, et al. The effect of intensive treatment of diabetes on the development and progression of long-term complications in insulin-dependent diabetes mellitus. N Engl J Med 1993;329:977-986.

11. Bergenstal RM, Beck RW, Close KL, et al. Glucose management indicator (GMI): a new term for estimating A1C from continuous glucose monitoring. Diabetes Care 2018; 41:2275-2280.

12. Becker RH, Dahmen R, Bergmann K, et al. New insulin glargine 300 units $\cdot \mathrm{mL}^{-1}$ provides a more even activity profile and prolonged glycemic control at steady state compared with insulin glargine 100 units $\cdot \mathrm{mL}^{-1}$. Diabetes Care 2015;38:637-643.

13. Becker RH, Nowotny I, Teichert L, et al. Low within- and between-day variability in exposure to new insulin glargine 300 U/ml. Diabetes Obes Metab 2015;17:261-267.

14. Heise T, Hovelmann U, Nosek L, et al. Comparison of the pharmacokinetic and pharmacodynamic profiles of insulin degludec and insulin glargine. Expert Opin Drug Metab Toxicol 2015;11:1193-1201.

15. Heise T, Nosek L, Bottcher SG, et al. Ultra-long-acting insulin degludec has a flat and stable glucose-lowering effect in type 2 diabetes. Diabetes Obes Metab 2012;14:944-950.

16. Heise T, Norskov M, Nosek L, et al. Insulin degludec: lower day-to-day and within-day variability in pharmacodynamic response compared with insulin glargine $300 \mathrm{U} / \mathrm{mL}$ in type 1 diabetes. Diabetes Obes Metab 2017;19:1032-1039.

17. Bailey TS, Pettus J, Roussel R, et al. Morning administration of $0.4 \mathrm{U} / \mathrm{kg} /$ day insulin glargine $300 \mathrm{U} / \mathrm{mL}$ provides less fluctuating 24-hour pharmacodynamics and more even pharmacokinetic profiles compared with insulin degludec $100 \mathrm{U} / \mathrm{mL}$ in type 1 diabetes. Diabetes Metab 2018;44:15-21.

18. Rosenstock J, Cheng A, Ritzel R, et al. More similarities than differences testing insulin glargine 300 units $/ \mathrm{mL}$ versus insulin degludec 100 units/mL in insulin-naive type 2 diabetes: the randomized head-to-head BRIGHT trial. Diabetes Care 2018;41:2147-2154.

19. Philis-Tsimikas A, Stratton I, Nørgård Troelsen L, et al. Efficacy and safety of degludec compared to glargine 300 units/mL in insulin-experienced patients with type 2 diabetes: trial protocol amendment (NCT03078478). J Diabetes Sci Technol 2019;13:498-506.

20. Philis-Tsimikas A, Klonoff DC, Khunti K, et al. Risk of hypoglycaemia with insulin degludec versus insulin glargine U300 in insulin-treated patients with type 2 diabetes: the randomised, head-to-head CONCLUDE trial. Diabetologia 2020;63:698-710.

21. Del Prato S. How conclusive is the CONCLUDE trial? Diabetologia 2020;63:692-697.

22. Philis-Tsimikas A, Klonoff DC, Khunti K, et al. Reduced risk of hypoglycaemia and lower HbAlc with degludec compared to glargine U300 in insulin-treated patients with type 2 diabetes. Diabetologia 2019;62(Suppl 1):1-600.
23. Heller SR, Buse JB, Ratner R, et al. Redefining hypoglycemia in clinical trials: validation of definitions recently adopted by the American Diabetes Association/European Association for the Study of Diabetes. Diabetes Care 2020; 43:398-404.

24. Home P. How valid are the new hypoglycemia definitions for use in clinical trials? Diabetes Care 2020;43:272-274.

25. Bergenstal RM, Bailey TS, Rodbard D, et al. Comparison of insulin glargine 300 units $/ \mathrm{mL}$ and 100 units $/ \mathrm{mL}$ in adults with type 1 diabetes: continuous glucose monitoring profiles and variability using morning or evening injections. Diabetes Care 2017;40:554-560.

26. Jinnouchi H, Koyama M, Amano A, et al. Continuous glucose monitoring during basal-bolus therapy using insulin glargine $300 \mathrm{U} \mathrm{mL} \mathrm{(-1)} \mathrm{and} \mathrm{glargine} 100 \mathrm{U} \mathrm{mL} \mathrm{(-1)} \mathrm{in}$ Japanese people with type 1 diabetes mellitus: a crossover pilot study. Diabetes Ther 2015;6:143-152.

27. Okajima F, Nakamura Y, Yamaguchi Y, et al. Basal-bolus insulin therapy with Gla-300 during hospitalization reduces nocturnal hypoglycemia in patients with type 2 diabetes mellitus: a randomized controlled study. Diabetes Ther 2018;9:1049-1059.

28. Galasso S, Facchinetti A, Bonora BM, et al. Switching from twice-daily glargine or detemir to once-daily degludec improves glucose control in type 1 diabetes. An observational study. Nutr Metab Cardiovasc Dis 2016;26:1112-1119.

29. Iga R, Uchino H, Kanazawa K, et al. Glycemic variability in type 1 diabetes compared with degludec and glargine on the morning injection: an open-label randomized controlled trial. Diabetes Ther 2017;8:783-792.

30. Iwasaki S, Kozawa J, Kimura T, et al. Insulin degludec is associated with less frequent and milder hypoglycemia in insulin-deficient patients with type 1 diabetes compared with insulin glargine or detemir. Diabetol Int 2017;8:228236.

31. Henao-Carrillo DC, Munoz OM, Gomez AM, et al. Reduction of glycemic variability with degludec insulin in patients with unstable diabetes. J Clin Transl Endocrinol 2018;12:8-12.

32. Yamamoto C, Miyoshi H, Fujiwara Y, et al. Degludec is superior to glargine in terms of daily glycemic variability in people with type 1 diabetes mellitus. Endocr J 2016;63: 53-60.

33. Thalange N, Deeb L, Iotova V, et al. Insulin degludec in combination with bolus insulin aspart is safe and effective in children and adolescents with type 1 diabetes. Pediatr Diabetes 2015;16:164-176.

34. Novo Nordisk. Data on file_Clinical study report_NCT0100 6291. http://filehosting.pharmacm.com/DownloadService .ashx?client=CTR_NVO_7271\&studyid=1229\&filename $=$ 3668-ctr-redacted.pdf (accessed June 13, 2019).

35. Novo Nordisk. Data on file_Clinical study report_NCT00 981244. http://filehosting.pharmacm.com/DownloadService .ashx?client=CTR_NVO_7271\&studyid=1222\&filename= 3579-ctr-redacted.pdf (accessed June 13, 2019).

36. Novo Nordisk. Data on file_Clinical study report_NCT00 982228. http://filehosting.pharmacm.com/DownloadService .ashx?client=CTR_NVO_7271\&studyid=1225\&filename $=$ 3583-ctr-redacted.pdf (accessed June 13, 2019).

37. ClinicalTrials.gov. A trial comparing the efficacy of insulin degludec with insulin glargine on glycaemic control using continuous glucose monitoring in patients with type 1 diabetes. https://clinicaltrials.gov/ct2/show/study/NCT01569841 (accessed December 15, 2019). 
38. Kawaguchi Y, Sawa J, Sakuma N, Kumeda Y. Efficacy and safety of insulin glargine $300 \mathrm{U} / \mathrm{mL}$ vs insulin degludec in patients with type 2 diabetes: a randomized, open-label, cross-over study using continuous glucose monitoring profiles. J Diabetes Investig 2019;10:343-351.

39. Yamabe M, Kuroda M, Hirosawa Y, et al. Comparison of insulin glargine $300 \mathrm{U} / \mathrm{mL}$ and insulin degludec using flash glucose monitoring: a randomized cross-over study. J Diabetes Investig 2019;10:352-357.

40. Battelino T, Bosnyak Z, Danne T, et al. InRange: comparison of the second-generation basal insulin analogues glargine $300 \mathrm{U} / \mathrm{mL}$ and degludec $100 \mathrm{U} / \mathrm{mL}$ in persons with type 1 diabetes using continuous glucose monitoring-study design. Diabetes Therapy 2020. [Epub ahead of print]; DOI: 10.1007/s13300-020-00781-6.

41. Ratner RE, Gough SC, Mathieu C, et al. Hypoglycaemia risk with insulin degludec compared with insulin glargine in type 2 and type 1 diabetes: a pre-planned meta-analysis of phase 3 trials. Diabetes Obes Metab 2013;15:175-184.

42. Ritzel R, Roussel R, Bolli GB, et al. Patient-level metaanalysis of the EDITION 1, 2 and 3 studies: glycaemic control and hypoglycaemia with new insulin glargine $300 \mathrm{U} / \mathrm{ml}$ versus glargine $100 \mathrm{U} / \mathrm{ml}$ in people with type 2 diabetes. Diabetes Obes Metab 2015;17:859-867.

43. Komuro M, Inoue $\mathrm{G}$, Tabata $\mathbf{M}$, et al. Insulin degludec requires lower bolus insulin doses than does insulin glargine in Japanese diabetic patients with insulin-dependent state. J Diabetes Sci Technol 2015;9:632-638.

44. Onda Y, Nishimura R, Ando K, et al. Comparison of glycemic variability in Japanese patients with type 1 diabetes receiving insulin degludec versus insulin glargine using continuous glucose monitoring: a randomized, cross-over, pilot study. Diabetes Res Clin Pract 2016;120:149-155.

45. Takahashi H, Nishimura R, Onda Y, et al. Comparison of glycemic variability in Japanese patients with type 1 diabetes receiving insulin degludec versus insulin detemir using continuous glucose monitoring: a randomized, cross-over, pilot study. Expert Opin Pharmacother 2017;18:335-342.

46. Tosaka Y, Kanazawa A, Ikeda F, et al. Switching from twice-daily basal insulin injections to once-daily insulin degludec injection for basal-bolus insulin regimen in Japanese patients with type 1 diabetes: a pilot study. Int $\mathbf{J}$ Endocrinol 2015;2015:176261.

47. Kuroda A, Tsuruo M, Aki N, et al. A pilot study comparing the CGM-assessed glycemic profiles of patients with type 1 diabetes on insulin degludec and insulin glargine. Diabetol Int 2017;8:112-115.
48. American Diabetes Association. 6. Glycemic targets: standards of medical care in diabetes-2019. Diabetes Care 2019;42(Suppl 1):S61-S70.

49. Fonseca VA, Grunberger G, Anhalt H, et al. Continuous glucose monitoring: a consensus conference of the American Association of Clinical Endocrinologists and American College of Endocrinology. Endocr Pract 2016;22:1008-1021.

50. Juvenile Diabetes Research Foundation Continuous Glucose Monitoring Study Group, Tamborlane WV, Beck RW, et al. Continuous glucose monitoring and intensive treatment of type 1 diabetes. N Engl J Med 2008;359:1464-1476.

51. Peters AL, Ahmann AJ, Battelino $T$, et al. Diabetes technology-continuous subcutaneous insulin infusion therapy and continuous glucose monitoring in adults: an Endocrine Society Clinical Practice Guideline. J Clin Endocrinol Metab 2016;101:3922-3937.

52. Danne $\mathrm{T}$, Nimri R, Battelino $\mathrm{T}$, et al. International consensus on use of continuous glucose monitoring. Diabetes Care 2017;40:1631-1640.

53. Tyndall V, Stimson RH, Zammitt NN, et al. Marked improvement in HbA1c following commencement of flash glucose monitoring in people with type 1 diabetes. Diabetologia 2019;62:1349-1356.

54. Charleer S, De Block C, Van Huffel L, et al. Quality of life and glucose control after 1 year of nationwide reimbursement of intermittently scanned continuous glucose monitoring in adults living with type 1 diabetes (FUTURE): a prospective observational real-world cohort study. Diabetes Care 2020;43:389-397.

55. Kusunoki Y, Katsuno T, Miyakoshi K, et al. Effects of switching from insulin glargine or detemir to insulin degludec in patients with type 1 diabetes mellitus. Diabetes Ther 2013;4:461-472.

56. Nakae R, Kusunoki Y, Katsuno T, et al. Medium-term effects of insulin degludec on patients with type 1 diabetes mellitus. Drugs R D 2014;14:133-138.

Address correspondence to: Tadej Battelino, MD

Professor of Pediatrics

UMC - University Children's Hospital

Bohoriceva 20

1000 Ljubljana

Slovenia

E-mail: tadej.battelino@mf.uni-lj.si 